\title{
High Sensitivity Magnetic Field Sensor for Spatial Applications
}

\author{
Amandine Bocheux \\ and Claude Cavoit \\ LPC2E \\ CNRS \\ Orléans, France \\ Email: cavoit@cnrs-orleans.fr
}

\author{
Myckael Mouchel \\ and Clarisse Ducruet \\ Crocus Technology \\ Grenoble, France \\ Email:mmouchel@crocus-technology.com
}

\author{
Romain Fons \\ Philippe Sabon \\ Ioan-Lucian Prejbeanu \\ and Claire Baraduc \\ UGA Grenoble - SPINTEC \\ UMR 8191 CEA/CNRS/UJF/G-INP \\ Grenoble, France \\ Email: claire.baraduc@cea.fr
}

\begin{abstract}
A high sensitivity 1D magnetic field sensor is developed for spatial applications, in order to replace the heavy search-coils currently used. This new sensor combines a flux concentrator, biasing coils for field modulation and magnetic tunnel junctions. These three elements are fabricated and independently characterized. Finally, the expected performance of a sensor combining these three elements can be estimated.
\end{abstract}

\section{INTRODUCTION}

Currently, space missions [1] are using inductive magnetic sensors with detectivity of less than a few tens of femtoTesla. Unfortunately, these sensors are large and relatively heavy (150g per axis), which is detrimental for the launching cost [2]. We propose an innovative ultra-sensitive vectorial magnetic sensor with similar sensitivity to current sensors but allowing a weight reduction of at least two orders of magnitude. The proposed spintronic sensor includes a magnetic circuit acting as a flux concentrator, biasing coils for field modulation and low noise magnetic tunnel junctions [3]. Its high sensitivity is obtained thanks to a strong amplification $(\approx 800)$ of the measured magnetic field by the flux concentrator. Moreover, the sensor noise is reduced by modulating the measured magnetic field by an ac biasing field, so that the sensor operating frequency is shifted beyond the range of $1 / \mathrm{f}$ noise. These features should allow a high sensitivity at frequencies below $10 \mathrm{kHz}$, with much better performances than the best current magnetoresistive sensors. Furthermore, the sensor small size increases its spatial resolution, which extends the scope of applications towards the medical sector, biotechnologies or non-destructive control, for example. Nevertheless, fabrication of this sensor requires to overcome many technical challenges. Therefore, the three main elements of the sensor (flux concentrator, biasing coils and magnetic tunnel junctions) are independently fabricated and characterized. In this paper, after introducing the working principle of the sensor, we describe the fabrication and characterization of the biasing coils and flux concentrator. Then we show the results obtained on the magnetic tunnel junctions used as unipolar devices. Finally, we discuss the expected sensitivity and detectivity of a complete sensor combining the three elements.

\section{WORKING PRINCIPLE}

A schematics of the sensor is presented in Fig. 1. A magnetic tunnel junction (MTJ) is inserted within the gap of a flux concentrator. Its fundamental state at zero field is the parallel configuration. When a magnetic field is applied perpendicularly to the anisotropy axis, the free layer magnetization rotates, leading to a unipolar response. In absence of external field, the junction is only subjected to the ac field of the biasing coils: so it produces a rectification of the bias signal. The frequency of the junction response is thus twice the ac field frequency (Fig. 1-a). When the dc field to measure is applied and subsequently amplified by the flux concentrator, it induces an offset of the oscillatory field produced by the biasing coils. For half a period, dc and ac fields add up, whereas for the next half period, both fields subtract, leading to a succession of larger and lower signals in the junction response (Fig. 1-b). By comparing the junction signals at successive half periods, the external dc field can be measured. This is performed thanks to a differential measurement of two junctions, which are biased with ac fields in antiphase.

\section{BIASING COILS}

Modulation of the magnetic field at high frequency allows to operate the magnetic tunnel junction beyond the frequency range of $1 / \mathrm{f}$ noise. In our sensor, modulation of the magnetic field is performed thanks to biasing coils producing a RF field at $100 \mathrm{kHz}$. An alternative technique is to use a micromechanical resonator to obtain a field modulation at a few $10 \mathrm{kHz}$ [4]. The biasing coils are defined as planar rectangular coils embedded in the substrate, underneath the flux concentrator. Their position with respect to the flux concentrator has been optimized to obtain the best efficiency. Their geometry results from a compromise between the coil efficiency, the coil resistance that must be kept low with respect to power consumption and the fact that the coil inner contact must not be below the flux concentrator for practical microfabrication issues. For achieving this last requirement, the wire width and pitch are smaller on the side of the rectangular coil, which extends outside the flux concentrator (Fig. 2). Fabrication of planar coils embedded in silicon oxide is realized by dry etching, 


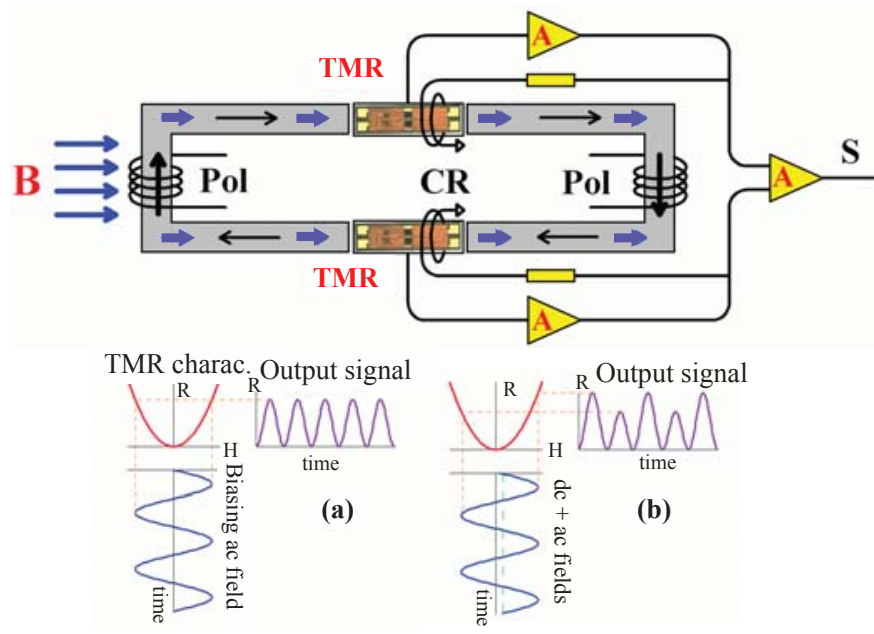

Fig. 1. Top: schematics of the sensor showing the magnetic tunnel junctions (TMR) within the gap of the flux concentrator, and the biasing coils (Pol); the short thick arrows represent the induction in the flux concentrator, due to the external field, and the long thin arrows the one due to the biasing coils. Bottom: a) output signal of the junction only subjected to the biasing ac magnetic field (zero external field); b) output signal of the junction when an external dc magnetic field is applied to the sensor

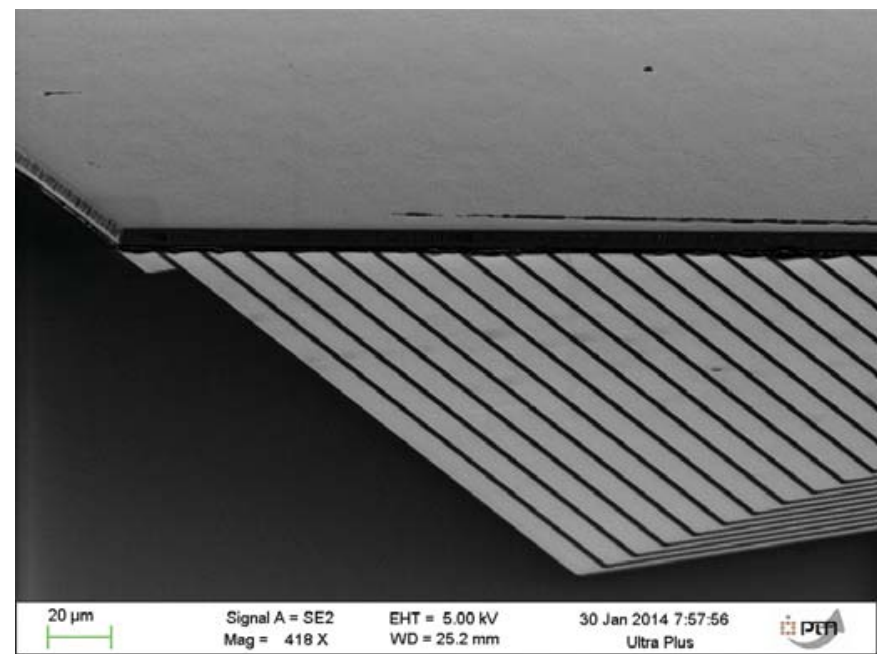

Fig. 2. View of the biasing coils extending outside the flux concentrator: the coils are embedded in the $\mathrm{SiO}_{2}$ substrate.

metal evaporation and lift-off. The silicon oxide thickness is thick enough to avoid any capacitive coupling between coils. This issue was checked by impedance measurements of the coils, that show a purely resistive behavior up to $1 \mathrm{MHz}$.

\section{FluX CONCENTRATOR}

The gap size of the flux concentrator is as small as possible to obtain a large gain. In order to keep the microfabrication process compatible with optical lithography and taking into account subsequent alignment steps, the gap size is fixed at $5 \mu \mathrm{m}$. Magnetic simulations show that the gain is about 800 for this gap size and drops rapidly when the gap broadens. Moreover the flux concentrator must be thick $(5 \mu \mathrm{m})$ in order to guide the modulation field produced by the planar biasing

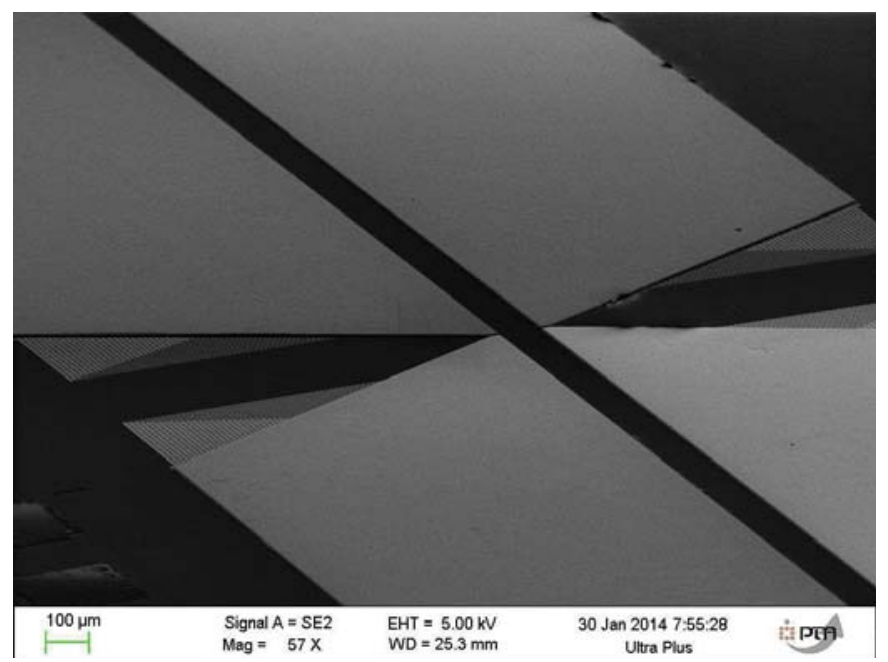

Fig. 3. View of the flux concentrator fabricated by electrodeposition in prepatterned resist.

coils. Fabrication of such a thick structure, with a gap of the same size as the film thickness, is quite challenging. The thick NiFe layer is obtained by electrodeposition in a resist mould made beforehand by photolithography of a thick resist layer (Fig 3). Magnetic measurements are then performed with a Vibrating Sample Magnetometer (VSM). The coercivity of the $\mathrm{NiFe}$ flux concentrator is found not to exceed $0.1 \mathrm{mT}$. The gain of the flux concentrator still needs to be checked by measuring the response of a tunnel junction before and after fabrication of the flux concentrator. We expect a larger gain compared to other studies that show a gain from 10 to 100 [5], [6], thanks to the narrow gap and the much higher thickness of our flux concentrator.

\section{Magnetic Tunnel Junctions}

\section{A. Junction Fabrication}

The junction stack was grown by conventional dc magnetron sputtering, starting form thermally oxidized Si substrates, on a Singulus Technologies Timaris cluster tool. The base pressure was $710^{-9}$ mbar and all depositions were performed at room temperature. The lower electrode contains a thick buffer layer (Ta $3 \mathrm{~nm} / \mathrm{CuN} 30 \mathrm{~nm} / \mathrm{Ta} 5 \mathrm{~nm}$ ) playing the role of an electrical contact, and a pinned synthetic antiferromagnet (PtMn 20 nm/ CoFe $2 \mathrm{~nm} / \mathrm{Ru} 0.8 \mathrm{~nm} / \mathrm{CoFeB} 2 \mathrm{~nm}$ ) as reference layer. The $\mathrm{MgO}$ tunnel barrier was obtain by deposition of $\mathrm{Mg}$ and subsequent plasma oxidation. The oxidation was performed by exposing the $\mathrm{Mg}$ metallic layer to a $40 \mathrm{~s}$ radiofrequency oxygen plasma at a pressure of 0.13 mbar and a rf power of $100 \mathrm{~W}$. The deposition of $1.1 \mathrm{~nm} \mathrm{Mg}$ layer followed by oxidation was repeated twice to ensure, after annealing, a $\mathrm{MgO}$ tunnel barrier with a resistance area product of 1.2 $\mathrm{k} \Omega \cdot \mu \mathrm{m}^{2}$ at room temperature. Finally, the upper electrode is a free layer of $\mathrm{CoFeB}$ protected by a capping layer (Ta $5 \mathrm{~nm} /$ $\mathrm{Ru} 7 \mathrm{~nm}$ ). In order to reduce the junction noise, the free layer thickness is large, ranging from 20 to $80 \mathrm{~nm}$. After deposition, the stack is annealed at $340^{\circ} \mathrm{C}$ under magnetic field to obtain 


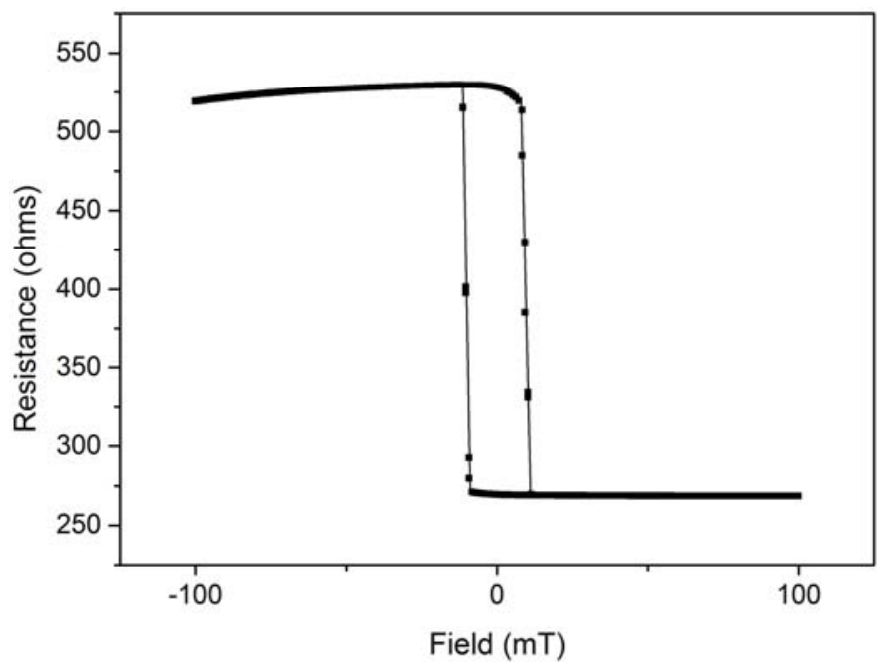

Fig. 4. Resistance versus field cycle for two $0.8 \times 8 \mu \mathrm{m}^{2}$ junctions $(20 \mathrm{~nm}$ thick free layer) connected in series, with magnetic field applied along the easy axis. The cycle is centered on zero field and the TMR is close to $100 \%$.

the proper magnetic alignment of the reference layer. It is then patterned into junctions by using deep UV lithography and ion-beam etching. Then electrical isolation and planarization are achieved by using Accuflo resist before processing the top electrode. An additional technological step may be required for the design of vias contacting the bottom electrode, before patterning the electrical contacts. Several types of rectangular junctions are thus obtained with different sizes: $0.8 \times 25 \mu \mathrm{m}^{2}$ (type I), $0.8 \times 8 \mu \mathrm{m}^{2}$ (type II), $0.8 \times 5 \mu \mathrm{m}^{2}$ (type III) and $0.8 \times 1.2 \mu \mathrm{m}^{2}$ (type IV).

\section{B. Easy Axis Characterization}

Resistance vs magnetic field cycles (R-H) have been measured with field applied along the easy axis. Sharp transitions are observed in Fig. 4. R-H cycles present a small offset, with an exchange field below $2 \mathrm{mT}$, due to a small uncompensation of the synthetic antiferromagnet. The low resistance state $\left(R_{P}\right)$, which corresponds to the parallel configuration of the free and reference layers magnetizations, is perfectly stable when increasing field. By contrast, in the antiparallel state, $R_{A P}$ resistance slightly decreases at large fields: this behavior is interpreted as the beginning of a spin-flop transition of the reference layer. The tunnel magnetoresistance ratio (TMR) is observed to increase as the junction size decreases: it is about $50 \%$ in the largest junctions and larger than $100 \%$ in the others, reaching even 120 to $140 \%$ in the smallest ones. The lower TMR value observed in large junctions is attributed to a serial interfacial resistance.

\section{Hard Axis Characterization}

As this sensor uses magnetic tunnel junctions operating with unipolar response, it is important to study the junction response when magnetic field is applied along the hard axis. Typical R-H cycle present the egg shape shown in Fig. 5. The low resistance curve with upward curvature corresponds

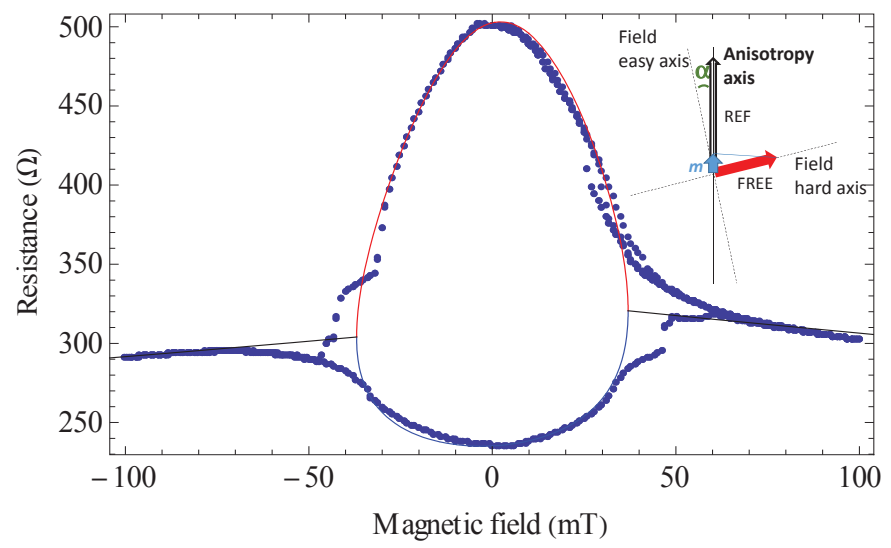

Fig. 5. Resistance versus field cycle (dots) for two $0.8 \times 8 \mu \mathrm{m}^{2}$ junctions (20 nm thick free layer) connected in series, with magnetic field applied along the hard axis. The fit (line) is performed assuming a coherent rotation of the free layer magnetization; the model takes into account the small rotation of the reference layer magnetization and the field slight misalignment $(\alpha)$. The fit parameters are: $H_{s}=37 \mathrm{mT}, H_{r}=500 \mathrm{mT}$, $\sin \alpha=0.075$, which correspond to a rotation of the reference magnetization of $4.2^{\circ}$ when the free layer magnetization has rotated by $90^{\circ}$ and a field misalignment angle of $\alpha=4.3^{\circ}$. Inset: schematics showing the field misalignment $(\alpha)$ with respect to anisotropy axis, and the small component, $m$, along the anisotropy axis of the free layer magnetization, at saturation field.

to the rotation of the free layer magnetization, from the parallel state at zero field (low resistance state $R_{P}$ ) towards the perpendicular configuration at saturation field $H_{s}$. This curve is the TMR characteristics shown in Fig. 1. Similarly, the high resistance curve with downward curvature corresponds to the gradual evolution from the antiparallel state at zero field (high resistance state $R_{A P}$ ) towards the perpendicular configuration at saturation field.A complete field cycle results in the full resistance loop shown in Fig. 5, due to a slight misalignment $(\alpha)$ of the applied field with respect to the anisotropy axis. At saturation field, the free layer magnetization becomes aligned with the field direction, which is not exactly at $90^{\circ}$ with respect to the anisotropy axis: the free layer magnetization has thus a small non-zero component $m$ along the anisotropy axis. Therefore, when decreasing the field value down to zero, the free layer magnetization rotates either toward the P or AP state depending on the sign of $\mathrm{m}$ (see inset of Fig.5.This behavior is observed for the two largest junctions (type I and II) and for the type III junctions having the thinnest free layer $(20 \mathrm{~nm})$.

The saturation field of the free layer can be extracted from the R-H cycles. For a given junction shape, $H_{s}$ is observed to increase with the free layer thickness. And for a given free layer thickness, $H_{s}$ increases with the shape factor defined as the length to width ratio (Fig. 6). This trend can be explained by the increasing shape anisotropy. A simple model assuming a coherent rotation of the free layer magnetization gives the same trend and can even fit the data obtained with the $20 \mathrm{~nm}$ thick free layer junctions. At saturation field, the free layer magnetization has rotated by $90^{\circ}$ with respect to the reference layer: this perpendicular configuration is characterized by the mean conductance $\frac{G_{P}+G_{A P}}{2}$. This conductance corresponds to the resistance value $\bar{R}^{2}=\frac{2 R_{P} R_{A P}}{R_{P}+R_{A P}}$, that gets closer to $R_{P}$ as 


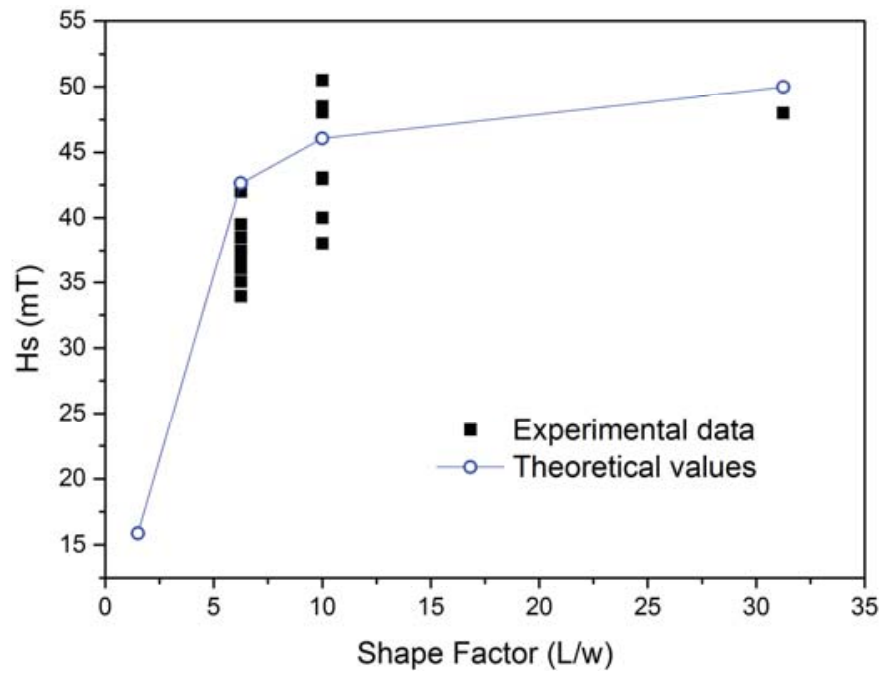

Fig. 6. Saturation fields obtained from the resistance versus hard axis field cycles, for junctions of different sizes, with $20 \mathrm{~nm}$ thick free layer. Data are plotted as a function of the length to width ratio of the junctions. The smallest junctions (type IV) have such distorted cycles that the saturation field cannot be extracted. The theoretical values are calculated assuming a coherent rotation of the free layer magnetization.

TMR increases. At fields higher than the saturation field $H_{s}$, the junction should remain in the perpendicular configuration with constant resistance. However we observe that the resistance slightly decreases, which is attributed to a small rotation of the reference layer magnetization. For ideally pinned reference layer, the junction behavior can be easily described. The junction conductance is $G=\frac{G_{P}+G_{A P}}{2}+\frac{G_{P}-G_{A P}}{2} \cos \theta$, where $\theta$ is the angle between the magnetizations of free and reference layers. In a macrospin description, the coherent rotation of the free layer magnetization is given by $\sin \theta=H / H_{s}=h$, where $H_{s}$ is the saturation field. Finally the conductance is equal to $G=\frac{G_{P}+G_{A P}}{2} \pm \frac{G_{P}-G_{A P}}{2} \sqrt{1-h^{2}}$, with positive (resp. negative) sign when starting from parallel (resp. antiparallel) state at zero field. However, to precisely describe the behavior of our junctions, we must take into account the fact that the reference layer is not perfectly pinned and slightly rotates at large field. Now the angle $\theta$ becomes the difference between the rotation angles of the free and reference layer magnetizations: $\theta=\theta_{F}-\theta_{R}$, where $\sin \theta_{F}=H / H_{s}$ and $\sin \theta_{R}=H / H_{r}$, with $H_{r}$ being the saturation field of the reference layer $\left(H_{r}>>H_{s}\right)$. Finally, the slight dissymmetry observed in the R-H curve between positive and negative fields can be explained by considering a small misalignment of the field with respect to the sample anisotropy axis. By taking all these parameters into account, a satisfactory fit of the experimental data can be obtained (Fig. 5). The misalignment turns out to be of the order of $4^{\circ}$ and the saturation field of the reference layer much larger than the one of the free layer: so, when the applied field reaches the free layer saturation field $H_{s}$, the reference layer magnetization has rotated by a small angle about $4^{\circ}$ to $10^{\circ}$ depending on the sample studied. This small rotation does not significantly change the hard-axis response of the tunnel junction. Nevertheless we plan to avoid this inconvenience by increasing the exchange biasing of the synthetic antiferromagnet.

\section{Noise Measurements}

Low frequency noise of the junctions has been measured as a function of the external field, applied either along the easy or the hard axis. The sample is contacted by probes inside a shielded environment. It is biased with a home-made current-like source composed of a battery with a large range potentiometer. The signal is then filtered by a high-pass filter $(-6 \mathrm{~dB}$ at $100 \mathrm{~Hz})$ and amplified by a low-noise pre-amplifier SR560. Then the signal is measured by a SR780 low frequency spectrum analyzer. The background noise, measured when the sample is not biased, averages $7 \mathrm{nV} / \sqrt{\mathrm{Hz}}$ at $1 \mathrm{kHz}$. The noise values presented below are corrected from the background noise and divided by the amplifier gain. Therefore, they do not include Johnson noise (estimated between 1 to $1.5 \mathrm{nV} / \sqrt{\mathrm{Hz}}$ for type I junctions). When the junction is in the parallel state, the measured noise does not exceed the background noise. In other magnetic configurations (antiparallel state or field sweep along the hard axis), all measured spectra show $1 / \mathrm{f}$ noise. In this case, the measured noise is essentially due to thermal excitations, since the shot noise is small, estimated around $2 \mathrm{nV} / \sqrt{\mathrm{Hz}}$ for type I junctions. Results obtained on a large rectangular magnetic tunnel junction $\left(0.8 \times 25 \mu \mathrm{m}^{2}\right)$ with $20 \mathrm{~nm}$ thick sense layer are presented in Fig. 7. The noise level is converted into Hooge factor [7], [8], to allow direct comparison with other studies: $\alpha_{H}\left(\mu m^{2}\right)=(A \times f) / V^{2} \times$ $S_{V}(H)$, where A is the junction area $\left(20 \mu \mathrm{m}^{2}\right), \mathrm{f}$ the frequency $(\approx 1 \mathrm{kHz}), \mathrm{V}$ the biased voltage $(0.1 \mathrm{~V})$ and $\mathrm{S}_{\mathrm{V}}(\mathrm{H})$ the noise under precise magnetic conditions. Fig. 7 presents the Hooge factor as a function of magnetic field, for different field sweeps. We observe a clear correlation between the resistance and the noise behavior as a function of magnetic field, as already observed in similar devices [9]. Noise is observed lower in parallel state compared to antiparallel state, and often higher when the field is applied along the hard axis. The Hooge coefficient is around $2.7 \times 10^{-10} \mu \mathrm{m}^{2}$ in antiparallel states, lower than $8 \times 10^{-11} \mu \mathrm{m}^{2}$ in parallel state (limited by the background noise). By comparison, Hooge factor is usually about $10^{-9} \mu \mathrm{m}^{2}$ in saturated states [10], [11]; nevertheless lower values such as a few $10^{-10} \mu \mathrm{m}^{2}$ were already reported in high quality tunnel junctions (for a review, see [12]). Moreover, noise at the working point along the hard axis is about $40 \mathrm{nV} / \sqrt{\mathrm{Hz}}$ at $1 \mathrm{kHz}$ which corresponds to a maximum Hooge factor of $3 \times 10^{-9} \mu \mathrm{m}^{2}$. This value is remarkably small compared to recent results showing a maximum Hooge factor of the order of $10^{-7} \mu \mathrm{m}^{2}$ [13], [14], probably linked to the high sensitivity of the junctions.

\section{EXPECTED PERFormanCES}

From the data we already obtained, the theoretical sensitivity and detectivity of the sensor can be estimated, assuming a satisfactory operation of the biasing coils and flux concentrator. By using the formula of the conductance as 


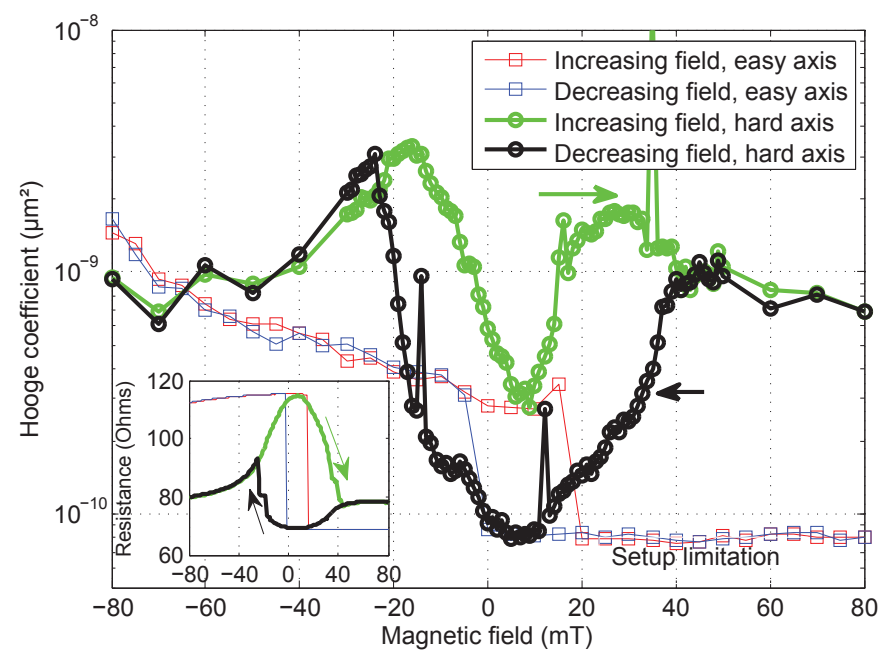

Fig. 7. Hooge factor as a function of applied field, for a large $0.8 \times 25 \mu \mathrm{m}^{2}$ single junction, with $20 \mathrm{~nm}$ thick free layer. Noise measurements are performed for field applied either along the easy or the hard axis. Inset: resistance versus field curves for field sweeps along easy and hard axis. A clear correlation is observed between these measurements and the noise level.

a function of the applied field (when reference is fixed), the junction sensitivity $s=\frac{1}{G_{0}} \frac{\mathrm{d} G}{\mathrm{~d} H}$ can be calculated, with $G_{0}$ being the conductance at the working point: $\frac{1}{G_{0}} \frac{\mathrm{d} G}{\mathrm{~d} H}=$ $\frac{1}{G_{0}} \frac{G_{P}}{2 H_{s}} \frac{\tau}{1+\tau} \frac{h}{\sqrt{1-h^{2}}}$, where $\tau$ is the TMR ratio. We notice that the sensitivity diverges when $H=H_{s}$. However, for experimental reasons, it is preferable not to work in the vicinity of the saturation, as we noticed some steps in the R-H curve close to $H_{s}$. So let us choose $H=0.85 H_{s}$ as a working point. Since the sensor sensitivity is equal to the junction sensitivity multiplied by the flux concentrator gain $\mathcal{G}$, its value at the working point is easily calculated $S \approx \mathcal{G} \frac{1.6}{H_{s}} \frac{\tau}{2+1.53 \tau}$. The sensor sensitivity can be numerically estimated, by using the experimental values of TMR and $H_{s}$ obtained for large junctions $(\mathrm{TMR}=50 \%, \mathrm{Hs}=50 \mathrm{mT})$ and the gain of the flux concentrator calculated by numerical simulations $(\mathcal{G}=800)$. With these values, we obtain a sensitivity of $460 \% / \mathrm{mT}$. We may also estimate the sensor detectivity, by taking into account the measured junction noise. Our experiments have shown that noise is of $1 / \mathrm{f}$ type and of about $40 \mathrm{nV} / \sqrt{\mathrm{Hz}}$ at $1 \mathrm{kHz}$ when magnetic field is applied along the hard axis. So we estimate a noise of $4 \mathrm{nV} / \sqrt{\mathrm{Hz}}$ at the modulating frequency $(100 \mathrm{kHz})$, which gives a detectivity of about $8.6 \mathrm{pT} / \sqrt{\mathrm{Hz}}$. Let us point out that this interesting performance is calculated assuming a perfect behavior of the modulated flux concentrator. It has to be checked experimentally when a complete sensor containing coils, flux concentrator and junctions will be fabricated. In particular, a crucial issue is the noise level of the junction under modulating field.

\section{CONCLUSION}

We developed a new type of ultra-sensitive magnetic field sensor for spatial applications. The sensor contains a flux concentrator, biasing coils and low-noise magnetic tunnel junctions. The latter work with the field applied along the hard axis leading to unipolar response. Each part of the sensor was fabricated and studied alone, to improve their properties. From the obtained data, the detectivity of a complete sensor is estimated about $8.6 \mathrm{pT} / \sqrt{\mathrm{Hz}}$, assuming a perfect behavior of the biasing coils and flux concentrator. Since the sensor sensitivity is inversely proportional to the saturation field, the sensor performance can be further improved by using a much softer free layer.

\section{ACKNOWLEDGMENT}

This work has been done thanks to the funding granted by CNES in the frame of a R\&T program in spintronics and by CNRS in the frame of a support to new and innovative sensor developments.

\section{REFERENCES}

[1] A. Roux, O. Le Contel, C. Coillot, A. Bouabdellah, B. de la Porte, D. Alison, S. Ruocco, and M.C. Vassal. The THEMIS mission. In Space Science Reviews, volume 141, pages 265-276. 2008.

[2] H C Séran and P Fergeau. An optimized low-frequency three-axis search coil magnetometer for space research. Review of Scientific Instruments, 76:44502, 2005.

[3] Claude Cavoit. Device for detecting and/or measuring a low magnetic field; International Patent Number WO 2010/109138 A1, 2010.

[4] A. Guedes, G. Jaramillo, C. Buffa, G. Vigevani, S. Cardoso, D. C. Leitao, P. P. Freitas, and D. A. Horsley. Towards picoTesla Magnetic Field Detection Using a GMR-MEMS Hybrid Device. IEEE Transactions on Magnetics, 48(11):4115-4118, 2012.

[5] R. C. Chaves, P. P. Freitas, B. Ocker, and W. Maass. MgO based picotesla field sensors. Journal of Applied Physics, 103, 2008.

[6] Z. Marinho, S. Cardoso, R. Chaves, R. Ferreira, L. V. Melo, and P. P. Freitas. Three dimensional magnetic flux concentrators with improved efficiency for magnetoresistive sensors. Journal of Applied Physics, 109(7):07E521, 2011.

[7] M. Fermon, C., Pannetier-Lecoeur. Noise in GMR and TMR Sensors. In Giant Magnetoresistance Sensors, volume 6, pages 47-70. Springer edition, 2013.

[8] F N Hooge. 1/f Noise. Physica B, 83:14-23, 1976.

[9] K. S. Kim, H. J. Shim, I. J. Hwang, B. K. Cho, J. H. Seok, and Jin Tae Kim. Magnetic field dependent noise in magnetic tunnel junction. Journal of Applied Physics, 91(10 I):8804-8806, 2002.

[10] W. F. Egelhoff, P. W T Pong, J. Unguris, R. D. McMichael, E. R. Nowak, A. S. Edelstein, J. E. Burnette, and G. a. Fischer. Critical challenges for picoTesla magnetic-tunnel-junction sensors. Sensors and Actuators A, 155:217-225, 2009.

[11] P P Freitas, R Ferreira, S Cardoso, and F Cardoso. Magnetoresistive sensors. Journal of Physics: Condensed Matter, 19:165221, 2007.

[12] Z. Q. Lei, G. J. Li, William F. Egelhoff, P. T. Lai, and Philip W T Pong. Review of noise sources in magnetic tunnel junction sensors. IEEE Transactions on Magnetics, 47(3):602-612, 2011.

[13] J. Y. Chen, J. F. Feng, and J. M. D. Coey. Tunable linear magnetoresistance in $\mathrm{MgO}$ magnetic tunnel junction sensors using two pinned $\mathrm{CoFeB}$ electrodes. Applied Physics Letters, 100, 2012.

[14] E. Paz, S. Serrano-Guisan, R. Ferreira, and P. P. Freitas. Room temperature direct detection of low frequency magnetic fields in the $100 \mathrm{pT} / \mathrm{Hz}^{\wedge} 0.5$ range using large arrays of magnetic tunnel junctions. Journal of Applied Physics, 115:17E501, 2014. 